\title{
A Qualitative Study of Undergraduates' Conceptualizations of Mental Illness
}

\author{
ELIZABETH MATTEO \\ Department of Psychology and Counseling, Alvernia University, \\ Reading, Pennsylvania, USA
}

\begin{abstract}
This qualitative study examined three stigma reduction interventions against mental illness stigma: education, video, and contact. Undergraduates $(\mathrm{N}=69)$ in three introductory psychology classes from a small, Catholic, liberal arts university in the northeast United States participated. Responses to two open-ended questions revealed common negative and stereotypical themes associated with mental illness. The benefits of supplementing traditional social distance measures with a qualitative approach, as well as the importance of considering a social developmental approach to stigma education are discussed.
\end{abstract}

KEYWORDS language, mental illness stigma, qualitative, stigma reduction

Over a decade has passed since the Surgeon General first reported stigma as the largest barrier to mental health treatment (U.S. Surgeon General, 1999). The report strongly emphasized that stigma must be combated to improve mental health treatment in the United States, indicating that it "deprives people of their dignity and interferes with their full participation in society" (p. 5). Significant areas of life in which personal freedoms may be severely limited or denied due to stigma include access and opportunity to adequate housing and employment, as well as fair treatment within the criminal justice system and the general healthcare system (Corrigan \& Kleinlein, 2005).

This research was partially funded by a Faculty Excellence Grant sponsored by Alvernia University. A poster that included the foundations of this research was presented at the 2011 American Association of Applied Linguistics, in Chicago, Illinois. I thank Roxana Delbene for her helpful comments during the preparation of this article.

Address correspondence to Elizabeth Matteo, Department of Psychology and Counseling, Alvernia University, 400 St. Bernardine St., Reading, PA 19607, USA. E-mail: Elizabeth.Matteo@ alvernia.edu 
Studies have also suggested that negative societal messages may become internalized in a process referred to as self-stigmatization (Gallo, 1994), which may lead to further negative repercussions or prevent individuals from seeking treatment at all (Corrigan \& Watson, 2002; Link \& Phelan, 2001). Data from the 2006 General Social Survey (GSS) indicates that the majority of the general U.S. population prefers to avoid social contact and perceives those with a mental illness as dangerous (Pescosolido et al., 2010).

Although mental illness stigma continues to be a problem, advocacy, education, and research efforts aimed at reducing stigma seem to have increased since the Surgeon General's 1999 Report. For instance, the National Alliance on Mental Illness has provided grassroots advocacy and education for several decades, and continues to expand programs and initiatives to improve the lives of individuals and families affected by mental illness. Bring Change 2 Mind, a national anti-stigma campaign, was founded in 2009. Their Web site information and public service announcements offer first-person perspectives from individuals and families managing mental illness. Additionally, the Chicago Consortium for Stigma Research (CCSR), developed in 2004 and supported by the National Institute of Mental Health, exclusively studies the stigma of mental illness. The impressive research efforts of the CCSR have been instrumental in providing a comprehensive framework for studying stigma that includes researchers from a variety of fields (Corrigan, 2005).

Stigma reduction research has tended to examine the effects of three types of strategies: contact, education, and protest (Corrigan \& Penn, 1999; Reinke, Corrigan, Leonhard, Lundin, \& Kubiak, 2004). Of these strategies, contact has generally been found to be the most effective. Factors that seem to reduce bias during direct contact include whether the individuals involved have equal status, work one-on-one, and engage cooperatively on tasks (Islam \& Hewstone, 1993; Kolodziej \& Johnson, 1996). Additionally, counterstereotypic members who are not perceived as an exception to the group may have a stronger influence on others' negative attitudes (Desforges et al., 1991). Even minimal contact may have a positive effect on attitudes. Reinke et al. (2004) found that 15 minutes of either videotaped or personal contact led to significant improvements on standard social distance measures, but only if the person disconfirmed stereotypes.

A range of methodologies has been employed to examine the nature and impact of mental illness stigma. In a review of mental illness studies, Link, Yang, Phelan, and Collins (2004) reported the largest percentage of articles (60.1\%) were non-experimental surveys, followed by experiments (16.2\%), qualitative studies (13.8\%), and review articles (11.4\%). They noted that qualitative studies seem to be underutilized and ranged widely in terms of how much depth they offered on the topic of stigma. Most qualitative studies focused on either media documents (e.g., news transcripts, print media, and movie depictions) or first person accounts from individuals living 
with mental illness in an effort to capture the nature of mental illness stigma. The relative lack of qualitative studies is surprising for several reasons. First, self-reported social distance measures may underestimate true bias because of social desirability. Moreover, predicting behavior from self-report can be problematic (Corrigan \& Penn, 1999; Krauss, 1995; Link et al., 2004). Finally, qualitative studies of media reports about mental illness illustrate a relationship between stigmatizing accounts of mental illness and negative public perceptions (Slopen, Watson, Gracia, \& Corrigan, 2007). This suggests the need to examine if interventions not only change individuals' self-reported social distance, but also influence the language people use to describe individuals diagnosed with a mental illness.

Recognizing the importance of stigma reduction, many educators have developed curriculum-based interventions targeted at secondary and postsecondary populations (Bizub \& Davidson, 2011; Chan, Mak, \& Law, 2009; Mann \& Himelein, 2008; Matteo \& You, 2012). Targeting people within educational settings may have several advantages compared to community-based advocacy efforts because they can be longer in length, require greater engagement, and provide more opportunity for ongoing discussion. Typical educational interventions have involved either personal contact or called on participants to engage with materials that exposed them to the perspective of individuals with mental illness (e.g., read narrative or watch a video). Chan et al. (2009) found that, in a sample of secondary students from Hong Kong, presenting a video following educational information was the most effective combination of interventions when compared to educational information alone or a video followed by educational information. In another study, reading first-person accounts from people with mental illness was effective at reducing stigma (Mann \& Himelein, 2008). Matteo and You (2012) found that a classroom activity in which introductory psychology students participated in small discussion groups with community members diagnosed with a mental illness was more effective than video or education alone. Finally, Couture and Penn (2006) examined how contact with people with mental illness over a 6-month period affected undergraduates' attitudes toward them. Students who volunteered for Compeer, a program designed to increase friendship between community volunteers and those with mental illness, showed a decrease in negative attitudes over time. Taken together, these studies provide evidence that various kinds of contact, structured within the educational context, may be effective at reducing stigma. Nonetheless, all of the studies relied primarily on self-reported social distance as the outcome measure.

In contrast, Bizub and Davidson (2011) used a qualitative approach by analyzing senior-level undergraduates' written responses to two questions about their experiences in a one-on-one contact intervention that was a semester long. Analyses of student responses suggested that despite having initial fears and anxiety concerning dangerousness and unpredictability, their 
previously held views were challenged through regular contact with their partners. Nonetheless, Bizub and Davidson (2011) noted that the students still entered the experience with many of the negative stereotypical beliefs held by the general public, despite having had many psychology courses that had targeted misconceptions about people with mental illness. Stigma reduction as a targeted student outcome may require designing and sequencing curricula in a way that considers prejudice reduction not only in terms of social psychological processes, but also as one that is developmental.

Building on prior research, the aims of this exploratory qualitative study were twofold: to examine themes drawn from students' responses about mental illness, and to compare the effects of education, video, and contact on students' associations, memories, and feelings about mental illness. Openended questions were developed and administered to elicit students' cognitive and affective associations about mental illness. It was hypothesized the students' responses in the contact condition would demonstrate less negative cognitive and affective conceptualizations at posttest compared to the other conditions.

\section{METHOD}

\section{Participants}

Participants in this study were all undergraduates attending a small, Catholic, liberal arts university in the northeast United States. A total of 69 students (41 women, $28 \mathrm{men}$ ) in three introductory psychology classes participated. Students were primarily freshman, non-psychology majors $(78 \% ; M$ age $=19$ years old; $S D=2.95$ ). Classes were drawn from a convenience sample, and were randomly assigned to the intervention conditions. Participation was completely voluntary and responses were coded to protect anonymity. All students chose to participate; however, only responses from participants completing both pre- and posttest assessments were used.

\section{Measures}

All three classes completed the same pre- and posttest questionnaires, which included demographic items (e.g., gender, major, year in school) and two vignettes describing "Bill" and "John," diagnosed with bipolar disorder and paranoid schizophrenia, which were used from Mann and Himelein (2004, 2008). Each vignette was followed by six social distance items derived from a 19-item social distance measure developed by Chung, Chen, and Liu (2001) and two open-ended items. The items read as follows: 1. When you hear about "mental illness" or "people who have a mental illness," what kind of associations can you make/come to mind? Write at least five. 2. When you 
hear about "mental illness" or people who have a mental illness," what kinds of feelings, perhaps memories, do these words/situations trigger for you? For the purposes of the current study, only the qualitative items were analyzed.

\section{Procedure}

After consenting to participate, students in all three classes completed the assessment 2 weeks prior to beginning the psychopathology unit. Instruction for the psychopathology unit directly corresponded to content in the textbook and publisher slides for all three classes (Griggs, 2009). All groups received approximately 6 hours of total instruction, of which 1 hour and 45 minutes was dedicated to the topic of mental illness stigma. For all groups, the intervention occurred after they had approximately 1 hour of class introducing them to the criteria used to establish psychopathology, the background and purposes of the Diagnostic and Statistical Manual of Mental Disorders (DSM-IV; American Psychiatric Association, 1994), and the advantages and disadvantages of diagnostic labels. The intervention phase involved the last 15 minutes of the first class and the entire following class (90 mins). During the remaining classes, students continued to learn about symptoms and diagnosis of various disorders and types of treatments. A week after completing the unit, instructors administered a 20 -item multiple-choice format psychopathology chapter quiz. Approximately 2 weeks after the psychopathology unit, all groups completed the posttest measure.

\section{Interventions}

The education intervention began by having students generate cultural stereotypes and myths about individuals with mental illness. During the following class (90 mins in length), the lecture and discussion focused on stereotypes and misconceptions associated with mental illness. The information challenged the causes, timeline, consequences, controllability, and common representations of mental illness often viewed in the media using an evidence-based approach.

The video group also began by engaging in a small group activity for 15 minutes. However, they generated questions they would ask a person with a mental illness. The instructor encouraged students to be honest and consider what they would be interested in knowing about the person's experiences. Students then completed an out-of-class assignment in which they had to view three public service announcements produced by a nonprofit organization whose mission is to reduce stigma surrounding mental illness. The Web site (http://www.bringchange2mind.org) featured firsthand accounts about mental illness. Afterward, students completed a short 1-1.5 page reflection paper addressing the following questions: "1. How can we as a 
society reduce the stigma associated with mental illness? 2. Do you think the website is effective? Support your answer." In an effort to control exposure time, students were told that the total assignment should take them no longer than 90 minutes to complete.

The contact group also engaged in the same 15-minute small group activity as the video group. However, in the following class period ( $90 \mathrm{~min}$ ), students met three community members diagnosed with a mental illness (diagnoses included schizophrenia, major depression, and attention deficit/ hyperactivity disorder). All were middle-aged men affiliated with a local mental health advocacy group. Each spent approximately 5 minutes (15 mins total) providing background about their lives (e.g., age of diagnosis, how they manage their illness, occupation, and family information). Students then sat with their groups from the previous class activity and the community members circulated to the groups for 15 minutes each. Every group had an opportunity to meet and ask questions of each community member. This procedure enabled more personal contact than a panel discussion, but might be considered less intimidating and more developmentally appropriate for lower level college students as compared to having a one-on-one conversation with a person whom they had never met.

\section{RESULTS}

\section{Associations}

Two coders worked independently to code the responses of the two items for themes. Six themes emerged for the first question: "When you bear about 'mental illness' or 'people who have a mental illness,' what kind of associations can you make/come to mind? Write at least five." Coders then independently recoded both the pre- and posttest responses. Given the higher proportion of female (59\%) to male students (41\%), as well as the variability in majors, these demographic variables were not factored into the analyses. The coder's interrater reliability was $91 \%$.

The six themes included: explanations for a mental illness diagnosis (e.g., "they have a chemical imbalance," "inherited problem"), attributions about people with mental illness (e.g., "unclear state of mind," "unpredictable," "slow"), the perceived needs of those with mental illness (e.g., "need to be monitored," "take medications to control illness," "can't be alone"), social representations associated with mental illness (e.g., "therapy," "asylum," "psychiatrist"), empathic reactions toward people (e.g., "judged by others," "get treated horribly"), and specific diagnosis (e.g., "depression," "anxiety," "retardation"). Instead of writing in complete sentences, the majority of responses were brief lists with one word or a phrase. Among the themes, attributions about people with mental illness reflected the greatest number of responses 
across all three groups at both pre- and posttest. Most of the attributions were negative, for example, common responses included: violent, crazy, socially awkward, unhappy, moody, gets distracted easily, and different. Empathic themes in which there was some attempt to take the perspective of the "other" or normalize those with mental illness made up less than 1\% of the total number of responses for the education and contact group at pretest. The video group had a higher percentage of empathic responses (12\%) at pretest. At posttest, the majority of empathic responses were found in the video (19\%) and contact groups (29\%). There was no change for the education group. Empathic posttest responses for the video and contact groups included: "they are just like everyone else except sometimes they need a little more care," and "they don't get the support they need... they are misunderstood."

For the contact condition, post-intervention responses also showed evidence of normalizing those with mental illness, for example, separate responses included the following phrases: can do anything anyone else can, usually can lead normal life, hard to pick out, recovery, and handling illness.

For all conditions, the pretest attributions, social representations, and diagnosis themes suggested students conflated mental illness with other developmental disorders, for example, "slow, retardation, special education, Down syndrome, Special Olympics, and autism" appeared in the responses. At posttest across conditions, there were fewer responses of this type, suggesting that learning about different diagnosis enabled students to better distinguish between mental and developmental disorders. The disorders listed at posttest reflected disorders more typically representing mental disorders, for example, "depression, schizophrenia, obsessive compulsive disorder, and bipolar."

\section{Memories and Feelings}

Students were also asked to describe the feelings or memories evoked by the words mental illness or person with a mental illness. Responses were first categorized as either a feeling or a memory. Within the category of feelings, themes that appeared with the greatest frequency included those expressing sadness, concern, and pity, discomfort and uncertainty, and feeling scared or fearful. For the category of memories, students' responses were further divided into those that were personal (i.e., about themselves, a friend, or a family member), related to school experiences (i.e., remembering experiences with other children or adolescents), and specific reference to movies they had seen ("A Beautiful Mind" or "Shutter Island").

Across all the groups, for both pre- and posttest, the feelings that appeared with the greatest frequency were those of sadness, sympathy, and concern. In the posttest contact condition, students' responses still indicated 
some of the same emotions (e.g., feeling sad or bad for people with mental illness); nonetheless, some responses revealed students' attempts to diminish the label, and how learning about possible behaviors that go along with illness is valuable.

I feel bad for them and want to help them. They are regular people just with a few problems and they deserve to live the same life we do.

Nothing really just that they have issues that make them unique. I feel that they are normal people.

I feel bad for them because labeling affects everyone, even me. It's hard not to judge a book by its cover. I think we need to start by not doing that.

It makes me feel sad, but it helps me to understand the types of behaviors they may show.

Some students disclosed personal experiences with mental illness, further highlighting the importance of raising awareness and addressing stigma in the context of undergraduate coursework on psychopathology. Below are three separate student responses:

They usually bring me back to the days when I was depressed and too anxious to even get out of bed in the morning in fear that my classmates would talk about me. I also think of my grandfather who has struggled with not only bipolar disorder but with PTSD as well, all related to the Vietnam War. I've seen him at his worst, in the hospital. And my mother is also one who has struggled with bipolar and it's heartbreaking to see what this disorder has done to her. My uncle has just been diagnosed with clinical depression and anxiety disorder. It's obvious that I've been surrounded by mental illness for most of my life, and in conclusion, it has affected me greatly.

Because I am also bipolar it makes me happy to know that I'm not alone. It's not just me, other people are going through similar things in their life.

When I hear about mental illness I immediately feel sad for the person because it is hard to live a normal life. Many memories flash in my mind when hearing the word schizophrenia. I immediately get scared because one of my family members is schizophrenic due to a chemical imbalance and many bad things happened because of it.

Other responses revealed how students' earlier school memories or experiences may have shaped their attitudes about mental illness. Several referenced students being segregated in the school setting. The responses below 
highlight how educational settings have the potential to perpetuate or change the status quo.

I think about the kids in my school who were always separate from us. They had different classes than we did and the only time we saw them was in the halls.

When I was in middle school the school was split in two, there was one floor specifically for the children with mental illness. I clearly remember how students in the school and even sometimes my own friends would make fun of them for not being the same or for not being able to "control" themselves.

It sometimes makes me feel bad because there are some people who make fun of people with mental illness. I remember when I was in middle school, one of my friends made fun of someone with a mental illness. It made me feel really bad and I corrected him by telling him that making fun of them only makes him look like a jerk.

Although response rates and variations in how students answered the second question made pre- and posttest comparisons across the groups difficult, one student in the contact condition reframed her memory of a negative high school experience after the intervention.

I had a situation occur with a schizophrenic boy in high school my freshman year-scary situation. I got pulled out of school for the day because of my safety. He had homicidal thoughts about others.

I still remember the kid from school who got expelled for violent behavior on grounds, but I realize now that that was an extreme circumstance and not everyone who has a mental illness acts that way.

The recognition that one negative instance cannot be generalized to all members of a group reflects an important progression in this students' thinking.

\section{DISCUSSION}

The findings from introductory psychology students' responses to two openended questions regarding their associations, feelings, and memories about mental illness before and after three stigma reduction interventions revealed consistent themes in how students communicate about mental illness. Preintervention, the most frequent associations were attributions about mental illness, followed by diagnosis, and social representations. At post-intervention, attributions remained the highest percentage of responses, the majority 
of which included negative stereotypes and misconceptions. Yet, the results also revealed that empathic responses increased for the video and contact conditions after the intervention. Disheartening as it may be to find students' attributions before and after intervention paralleled many misconceptions and stereotypes about mental illness, the fact that increases in empathy were reflected in students' language after a 1 hour, 45 minute educational intervention is promising and supplements the findings of quantitative research showing that contact interventions are effective at decreasing social distance. Moreover, students' responses within this study suggest that empathy is an important component in this process.

Feelings about mental illness most frequently described were sadness and pity, followed by discomfort and fear. This result is consistent with research showing that dimensions of warmth and competence are associated with different kinds of prejudices toward various out-groups, and that pity is most often associated with the elderly, disabled, and developmentally challenged (Fiske, Cuddy, Glick, \& Xu, 2004). These groups are seen as high on warmth, but low on competence. It would seem from this analysis that those with a mental health diagnosis are judged in a similar manner, which may also help explain why many of the pretest associations conflated developmental, cognitive, and mental disabilities. In addition to listing mood or psychotic disorders, students also wrote retardation, autism, Down's syndrome, and attributions such as "slow" and "toddler-like." Based on students' reported memories, the misconception that individuals with a mental illness may also have learning or intellectual impairments may develop from experiences in schools in which children with any exceptionality are segregated from the general population. In addition to schools being important contexts that shape students' conceptions, personal experiences and media were also found to be more salient memories.

\section{LIMITATIONS AND FUTURE DIRECTIONS}

Using the broad term mental illness in this study likely obscured some of the differences in how diagnostic labels are perceived. Other research indicates that disabilities carry varying attributions of controllability and stability. For example, physical disabilities are viewed as less controllable than other disabilities, and mental illness is often viewed as something unchangeable (Corrigan, 2007; Corrigan et al., 2001). These attributions are important because they can influence blame and avoidance. In addition to different diagnostic labels showing variation in controllability and stability attributions, terms such as "mental health consumer" and "recovery," which were used by the community members in the contact condition for this study, may play an important role in changing perceptions. Understanding how terms such as these may influence attributions of stability and control warrants 
further examination. In a similar vein, researchers exploring children's perceptions of mental health in Scotland intentionally used a health promotion approach and used "positive mental health" in their focus group discussions with adolescents (Armstrong, Hill, \& Secker, 2000). Future qualitative stigma reduction studies should consider how particular terms may impact responses.

Just as social desirability is a concern with traditional social distance ratings, it cannot be ruled out with written responses. However, in this study, students were surprisingly candid about communicating prejudices, which on one hand is disheartening because it indicates a lack of awareness of the unacceptability of negative attitudes and stereotypes about individuals with a mental illness; however, this also suggests responses were not influenced by social pressure to appear politically correct. Students also completed their responses during the last few minutes of class, which took approximately 10-15 minutes, so what they wrote was not based on a period of long contemplation. Depending on the aims of future qualitative research, the method of responding (i.e., written, oral) and timing should certainly be considered in the design.

Like other research involving contact interventions, the current study was relatively short term. Although short-term interventions are less time consuming, possibly less costly, and may show statistically significant change, from an educational standpoint it would be beneficial to think of stigma reduction as a social-developmental process. Bizub and Davidson (2011) found that college seniors had many fears and anxieties prior to befriending someone with a mental illness despite the fact that stigma had been addressed in their lower-level courses. Psychology and other related disciplines might consider stigma reduction as a multistep, developmental process in which particular outcomes are met at each stage. Following Mann and Himelein (2008), the impetus behind exposing introductory psychology students to stigma reduction interventions was based in a desire to reach a fairly large and varied student population. In terms of professional preparation for the undergraduate psychology major, structured contact interventions that occur earlier may be an effective way to reach the American Psychological Association's (2007) diversity outcomes related to student learning for undergraduate education (Goal 8) and could better prepare students for internship or entry-level positions in practical settings.

In conclusion, this qualitative study supplements previous research on stigma reduction techniques used in undergraduates. Students' written responses to questions about mental illness revealed negative misconceptions consistent with quantitative research. Analyses revealed that contact interventions may be an effective means for increasing empathy toward individuals with mental illness. Other major themes suggest that those with mental illness more often provoke feelings of sadness and pity. Finally, memories associated with mental illness were primarily derived from personal experiences, school, and movies. 


\section{REFERENCES}

American Psychiatric Association. (1994). Diagnostic and statistical manual of mental disorders (4th ed.). Washington, DC: Author.

American Psychological Association. (2007). APA guidelines for the undergraduate psychology major. Washington, DC: Author. Retrieved from http://www.apa. org/ed/precollege/about/psymajor-guidelines.pdf

Armstrong, C., Hill, M., \& Secker, J. (2000). Young people's perceptions of mental health. Children E Society, 14, 60-72.

Bizub, A. L., \& Davidson, L. (2011). Stigma-busting, Compeer and the psychology student: A pilot study on the impact of contact with a person who has a mental illness. The Humanistic Psychologist, 39, 312-323.

Chan, J. Y. N., Mak, W. W. S., \& Law, L. S. C. (2009). Combining education and videobased contact to reduce stigma of mental illness: "The same or not the same" anti-stigma program for secondary schools in Hong Kong. Social Science and Medicine, 68, 1521-1526.

Chung, K. F., Chen, E. Y. H., \& Liu, C. S. M. (2001). University students' attitudes towards mental patients and psychiatric treatment. International Journal of Social Psychiatry, 47, 63-72.

Corrigan, P. W. (Ed.). (2005). On the stigma of mental illness: Practical strategies for research and social change. Washington, DC: American Psychological Association.

Corrigan, P. W. (2007). How clinical diagnosis might exacerbate the stigma of mental illness. Social Work, 52, 31-39.

Corrigan, P. W., \& Kleinlein, P. (2005). The impact of mental illness stigma. In P. Corrigan (Ed.), On the stigma of mental illness: Practical strategies for research and social change (pp. 11-44). Washington, DC: The American Psychological Association.

Corrigan, P. W., \& Penn, D. L. (1999). Lessons from social psychology on discrediting psychiatric stigma. American Psychologist, 54, 765-776.

Corrigan, P. W., River, L. P., Lundin, R. K., Penn, D. L., Wasowski, K. U., Campion, J., ... Kubiak, M. A. (2001). Three strategies for changing attributions about severe mental illness. Schizophrenia Bulletin, 27, 187-196.

Corrigan, P. W., \& Watson, A. S. (2002). The paradox of self-stigma and mental illness. Clinical Psychology: Science and Practice, 9, 35-53.

Couture, S. M., \& Penn, D. L. (2006). The effects of prospective naturalistic contact on the stigma of mental illness. Journal of Community Psychology, 34, 635-645.

Desforges, D. M., Lord, C. G., Ramsey, S. L., Mason, J. A., VanLeeuwen, M. D.,West, S. A., \& Lepper, M. R. (1991). Effects of structured cooperative contact on negative attitudes toward stigmatized groups. Journal of Personality and Social Psychology, 60, 531-544.

Fiske, S. T., Cuddy, A. C., Glick, P., \& Xu, J. (2002). A model of (often mixed) stereotype content: Competence and warmth respectively follow from perceived status and competition. Journal of Personality \& Social Psychology, 82, 878-902.

Gallo, K. M. (1994). First person account: Self-stigmatization. Schizophrenia Bulletin, 20, 407-410. 
Griggs, R. A. (2009). Psychology: A concise introduction (2nd ed.). New York, NY: Worth.

Islam, M. R., \& Hewstone, M. (1993). Dimensions of contact as predictors of intergroup anxiety, perceived out-group variability, and out-group attitude: An integrative model. Personality and Social Psychology Bulletin, 19, 700-710.

Kolodziej, M. E., \& Johnson, B. T. (1996). Interpersonal contact and acceptance of persons with psychiatric disorders: A research synthesis. Journal of Consulting and Clinical Psychology, 64, 1387-1396.

Krauss, S. J. (1995). Attitudes and the prediction of behavior: A meta-analysis of the empirical literature. Personality and Social Psychology Bulletin, 21, 58-75.

Link, B. G., \& Phelan, J. C. (2001). Conceptualizing stigma. Annual Review of Sociology, 27, 363-385.

Link, B. G., Yang, L. H., Phelan, J. C., \& Collins, P. Y. (2004). Measuring mental illness stigma. Schizophrenia Bulletin, 30, 511-541.

Mann, C. E., \& Himelein, M. J. (2004). Factors associated with stigmatization of persons with mental illness. Psychiatric Services, 55, 185-187.

Mann, C. E., \& Himelein, M. J. (2008). Putting the person back in psychopathology: An intervention to reduce mental illness stigma in the classroom. Social Psychiatry and Psychiatric Epidemiology, 43, 545-551.

Matteo, E. K., \& You, D. (2012). Reducing mental illness stigma in the classroom. Teaching of Psychology, 39, 121-124.

Pescosolido, B., Martin, J., Long, S., Medina, T., Phelan, J., \& Link, B. (2010). "A disease like any other"? A decade of change in public reactions to schizophrenia, depression, and alcohol dependence. American Journal of Psychiatry, 167, 1321-1330.

Reinke, R. R., Corrigan, P. W., Leonhard, C., Lundin, R. K., \& Kubiak, M. A. (2004). Examining two aspects of contact on the stigma of mental illness. Journal of Social and Clinical Psychology, 23, 377-389.

Slopen, N. B., Watson, A. C., Gracia, G., \& Corrigan, P. W. (2007). Age analysis of newspaper coverage of mental illness. Journal of Health Communication, 12, $3-15$.

U.S. Surgeon General. (1999). Mental health: A report of the Surgeon General. Washington, DC: U.S. Department of Health and Human Services. Retrieved from http://www.surgeongeneral.gov/library/mentalhealth/toc.html 